\section{NEW PRODUCTS COMPLY WITH WHITENING RULES}

From 31 October 2012, all tooth whitening products are permitted to contain from $0.1 \%$ to $6 \%$ hydrogen peroxide and the supply of these products is restricted to registered dental professionals only. SDI's Pola Day 3\% and 6\% hydrogen peroxide and Pola Night 10\% and 16\% carbamide peroxide comply with the new whitening regulation and are now available.

SDI has successfully gained approval for the range of Pola products which

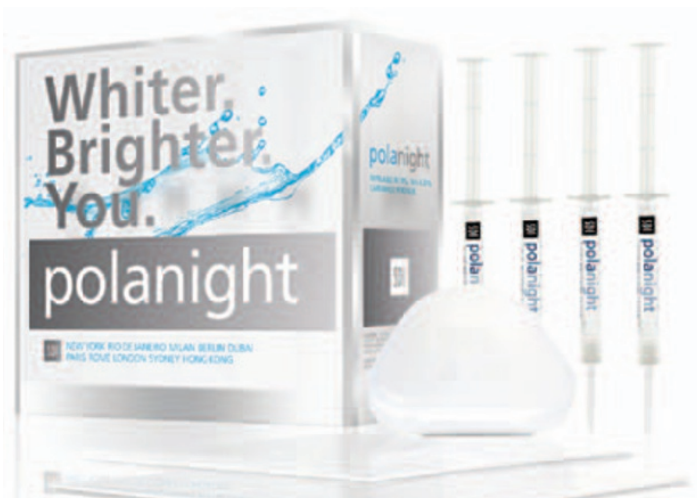

contain greater than $6 \%$ hydrogen peroxide as medical devices under a revised product classification. The packaging will comply with Medical Device Directive requirements based on modified 'Indications of Use' as follows: lightly etching away surface staining on vital teeth; lightly etching away surface staining on non-vital teeth; assisting in the brightening of discoloured teeth; and removal of surface stains on teeth discoloured by medications such as tetracycline, minocycline, fluorosis, food related stains and age dependent changes.

These products are: Pola Day 7.5\% and $9.5 \%$ hydrogen peroxide, Pola Night 22\% carbamide peroxide, Pola Day CP 35\% carbamide peroxide, Pola Office 35\% hydrogen peroxide and Pola Office Plus 37.5\% hydrogen peroxide.

These higher percentages will enable dental practices to continue to offer very fast treatments.

www.polawhite.com.au/

\title{
AN UNOBTRUSIVE WELCOME FOR PATIENTS
}

The design of the new tbCompass Treatment Centre from Takara Belmont is logical; with a delivery unit that can rotate behind the chair, it provides an easy and unobtrusive welcome for your patients. Nurses will also benefit from this feature as it provides the ideal position for essential clean and prep work.

The unique centrally mounted pivoting mechanism allows the tbCompass to convert easily from right to left handed use in less than 90 seconds, without the need for any tools. An ambidextrous unit is great for practices where a room is shared, or for those who want the flexibility in the future.

As well as optimising efficiency and performance, the chair is designed to be relaxing with an ergonomically designed lumbar support and twin articulating headrest to ensure patient comfort. The tbCompass also has a new luxury upholstery option, designed for those looking for extra comfort and elegance, whilst the standard seamless version is ideal for those who just want a pragmatic and comfortable option.

The tbCompass also benefits from Takara Belmont's free fiveyear extended warranty, offering additional peace of mind when purchasing capital equipment.

Call 02075150333 without obligation.

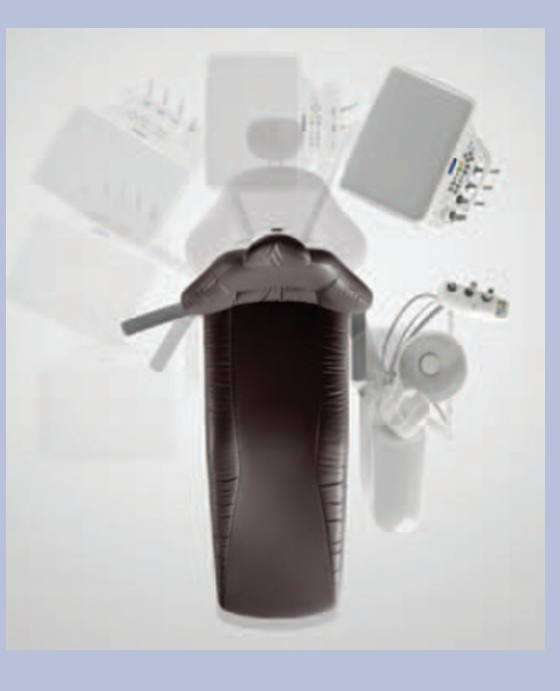

\section{RECORD YOUR PATIENTS'} BITE FORCE

US-based Tekscan, Inc. has launched a diagnostic device called the T-Scan 8 that records a patient's bite force and enables a dentist to determine a patient's BiteForce Dynamics, which includes occlusal force, timing and location. It features an all new user interface and workflow designed to optimise efficiency when evaluating occlusal forces.

The T-Scan is built with patented sensor technology and puts articulating marks into context by showing you where and how occlusal forces occur in a patient's bite.

Dentists all over the world are using the T-Scan to protect their cosmetic cases from harmful forces, ensuring proper implant loading and preventing remakes and fractures, minimising emergency visits, and saving valuable chair time.

The data recorded by the T-Scan put you in control of the treatment sequence by providing the percentage of force distribution for a patient's bite. There is no need to rely on 'How does that feel?' to qualify treatment.

For more information visit www. tekscan.com/dental or contact Tekscan at marketing@tekscan.com.

\section{TRIED, TESTED, TRUSTED}

Curaprox UK offers a whole host of tried and trusted oral hygiene products:

- Curasept ADS: chlorhexidine products with a unique non-staining formula; includes alcohol-free mouthwash and SLS-free toothpaste and gel

- Hydrosonic Electric Toothbrush: provides 42,000 sonic waves per minute for a brilliant and easy clean

- CPS Prime interdental brushes: last five times longer than any other interdental brush on the market

- IAP professional probing tool: determines ideal interdental brush size

- IAC personalised prescription: interdental recommendations.

Visit www.curaprox.co.uk. 